Canadian

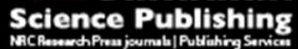

Canadian Journal of Physiology and Pharmacology Revue canadienne de physiologie et pharmacologie

\title{
A global profile of glucose-sensitive endothelial-expressed long non-coding RNAs
}

\begin{tabular}{|r|l|}
\hline Journal: & Canadian Journal of Physiology and Pharmacology \\
\hline Manuscript ID & cjpp-2015-0585.R1 \\
\hline Danuscript Type: & Article \\
\hline Complete List of Authors: & $\begin{array}{l}\text { Singh, Krishna; St Michael's Hospital } \\
\text { Mantella, Laura-Eve; St Michael's Hospital } \\
\text { Pan, Yi; St Michael's Hospital } \\
\text { Quan, Adrian; St Michael's Hospital, } \\
\text { Sabongui, Sandra; St Michael|'s Hospital } \\
\text { Sandhu, Paul; St Michael''s Hospital } \\
\text { Teoh, Hwee; St Michael's Hospital, } \\
\text { Al-Omran, Mohammed; St Michael's Hospital, } \\
\text { Verma, Subodh; St Michael's Hospital, Cardiac Surgery }\end{array}$ \\
\hline Keyword: & Incrna, human endothelial cells, elevated glucose \\
\hline
\end{tabular}

SCHOLARONE $^{\text {IM }}$

Manuscripts 


\section{A global profile of glucose-sensitive}

\section{endothelial-expressed long non-coding RNAs}

Krishna K. Singha,b,e, , Laura-Eve Mantellaa,d, , Yi Pana, Adrian Quana, Sandra Sabonguia, Paul Sandhu ${ }^{\mathrm{a}}$, Hwee Teoha,c, Mohammed Al-Omran ${ }^{\mathrm{b}, \mathrm{e}, \mathrm{f}}$, and Subodh Verma ${ }^{\mathrm{a}, \mathrm{d}, \mathrm{e} . \dagger}$

Divisions of aCardiac Surgery, bVascular Surgery, and cEndocrinology \& Metabolism, Keenan Research Centre for Biomedical Science and Li Ka Shing Knowledge Institute of St. Michael's Hospital, Toronto, ON, Canada; Departments of dPharmacology \& Toxicology and eSurgery, University of Toronto, Toronto, ON, Canada; Department of Surgery, King Saud University and the King Saud University-Li Ka Shing Collaborative Research Program, Riyadh, Kingdom of Saudi Arabia

*These authors contribute equally to this work.

†Corresponding address: Division of Cardiac Surgery, St. Michael's Hospital, $8^{\text {th }}$ Floor, Bond Wing, 30 Bond Street, Toronto, ON, M5B 1W8, Canada, Tel.: 1-416-864-5997; Fax: 1-4168645881; e-mail: vermasu@smh.ca

Word count: 2,431 


\section{ABSTRACT}

Hyperglycemia-related endothelial dysfunction is believed to be the crux of diabetesassociated micro- and macrovascular complications. We conducted a systematic transcriptional survey to screen for human endothelial long non-coding RNAs (lncRNAs) regulated by elevated glucose levels. LncRNAs and protein-coding transcripts from human umbilical vein endothelial cells (HUVECs) cultured under high (25 mmol/L) or normal (5 mmol/L) glucose conditions for 24 hours were profiled with the Arraystar Human LncRNA Expression Microarray V3.0. Of the 30,586 lncRNAs screened, 100 were significantly upregulated and 186 appreciably downregulated $(P<0.05)$ in response to high glucose exposure. In the same HUVEC samples, 133 of the 26,109 mRNAs screened were upregulated and 166 downregulated. Of these 299 differentially expressed mRNAs, 26 were significantly associated with 28 differentially expressed long intergenic non-coding RNAs $(P<0.05)$. Bioinformatics analyses indicated that the mRNAs most upregulated are primarily enriched in axon guidance signaling pathways; those most downregulated are notably involved in pathways targeting vascular smooth muscle cell contraction, dopaminergic signaling, ubiquitin-mediated proteolysis, and adrenergic signaling. This is the first lncRNA and mRNA transcriptome profile of high glucose-mediated changes in human endothelial cells. These observations may prove novel insights into novel regulatory molecules and pathways of hyperglycemia-related endothelial dysfunction and accordingly diabetes-associated vascular disease.

Abstract word count: 200

Keywords: lncRNA, human endothelial cells, elevated glucose 


\section{INTRODUCTION}

Long-term follow-up studies in large type 2 diabetes cohorts have demonstrated that diabetes-associated microvascular and macrovascular complications are directly related to the severity and duration of hyperglycemia (Hayward et al. 2015; Holman et al. 2008). A healthy endothelium is crucial to vascular health and anomalies in endothelial function, secondary to hyperglycemia (Avogaro et al. 2011), may underlie the clinical observations of elevated cardiovascular risk in individuals with diabetes relative to those without the disease (Booth et al. 2006). Recognized contributors of diabetes-associated endothelial dysfunction include protein kinase C (PKC), hexosamine and polyol pathways, and advanced glycation end-products (AGEs), all of which promote reactive oxygen species bioavailability and reduce nitric oxide production thereby altering endothelial cell structure and physiology (Giacco and Brownlee 2010; Shaw et al. 2014).

Long non-coding RNAs (lncRNAs), previously perceived as transcriptional noise due to their low expression and poor evolutionary conservation (Hangauer et al. 2013), have emerged as potential molecular determinants of a wide range of diseases. Similar to mRNAs, IncRNAs are transcribed by RNA-polymerase II, spliced, polyadenylated, and capped (Chew et al. 2013). They contain signature histone H3 on lysine 4 (H3K4) and Lys36 on histone (H3K36) trimethylation patterns that demarcate active promoters and transcribed regions (Fatica and Bozzoni 2014); and they can regulate nearby proteincoding genes in cis or those located further in trans (Rinn et al. 2007).

Transient hyperglycemia triggers gene-activating epigenetic changes and molecular cross-talks that are fundamental to the initiation and advancement of vascular anomalies (Cooper and El-Osta 2010). Morán and colleagues noted aberrant expression of the 
lncRNAs KCNQ1OT1 and HI-LNC45 in pancreatic islets from individuals with type 2 diabetes and upregulation of the long intergenic non-coding RNAs (lincRNAs) HI-LNC78 and HI-LNC80 in cultures of purified human pancreatic islets following exposure to a high glucose milieu (Moran et al. 2012). Another group also working with human pancreatic islets found significant associations between 17 lincRNAs and glycated hemoglobin levels (Fadista et al. 2014). Fibrovascular membranes of individuals with diabetes had higher levels of the lncRNA myocardial infarction-associated transcript (MIAT) than idiopathic epiretinal membranes from persons without diabetes (Yan et al. 2015). The notion that IncRNAs may regulate the diabetes-atherosclerosis axis is supported by reports that ANRIL, a lncRNA associated with risk for coronary atherosclerosis, carotid arteriosclerosis and peripheral artery disease, maps to an established type 2 diabetes susceptibility locus (Chen et al. 2014). The goal of this study was accordingly to address the potential that high glucose-associated changes in IncRNA expression may be fundamental to diabetesassociated vascular disorders. 


\section{MATERIALS AND METHODS}

Cell Culture. Human umbilical vein endothelial cells (HUVECs from pooled donors, Lonza) were cultured in EC growth medium-2 (EGMTM-2 BulletkitTM; Lonza) supplemented with growth factors, serum and antibiotics at $37{ }^{\circ} \mathrm{C}$ in humidified $5 \% \mathrm{CO}_{2}$. Confluent HUVECs were starved overnight before exposure to study conditions. HUVECs designated to the control (normal glucose) group were maintained for 24 hours in $5 \mathrm{mmol} / \mathrm{L}$ glucose and 20 mmol/L mannitol to account for changes that may be triggered by osmolarity differences. The remaining HUVECs were maintained for the same duration in glucose-enriched (final glucose concentration $25 \mathrm{mmol} / \mathrm{L}$ ) media.

Microarray Profiling. Total RNA was isolated from HUVECs using TRIzol reagent and quantified with the NanoDrop ND-1000 spectrophotometer. RNA integrity was confirmed by standard denaturing agarose gel electrophoresis. The expression profile of 30,586 human IncRNAs and 26,109 protein-coding transcripts was conducted with the Arraystar Human LncRNA Microarray V3.0. Sample labeling and array hybridization were performed on the Agilent Array platform. Briefly, total RNA from each sample was amplified and transcribed into fluorescent cRNA (Arraystar Flash RNA Labeling Kit, Arraystar) before 1 $\mu \mathrm{g}$ of each labeled cRNA was hybridized onto the microarray slide. The hybridized arrays were washed, fixed and scanned with the Agilent DNA Microarray Scanner (Product\# G2505C). The acquired array images were analyzed with the Agilent Feature Extraction software (version 11.0.1.1). Quantile normalization and subsequent data processing were performed with the GeneSpring GX v11.5.1 software package (Agilent Technologies). Volcano plot filtering, set at a threshold of $\geq 2.0$ folds, was used to screen for lncRNAs and 
mRNAs that exhibited significantly different $(P<0.05$; unpaired t-test adjusted for multiple testing with the Benjamini Hochberg method to minimize the false discovery rate) expression levels in the two study groups. Pathway analysis was based on the current Kyoto Encyclopedia of Genes and Genomes (KEGG) database. Gene Ontology (GO) analysis was performed with the topGO package of bioconductor system.

\section{qPCR Validation}

The levels of select lncRNAs in HUVECs cultured as described above were evaluated using standard qPCR methods. 


\section{RESULTS}

The 6 samples evaluated had a 2:1 intensity ratio for their 28S:18S rRNA bands and OD260/OD280 ratios of $>1.8$ thereby verifying RNA integrity, purity and concentration (Supplementary Fig. 1). Box-and-Whisker plots constructed to visualize the distribution of the fluorescent intensities revealed very similar normalized $\log 2$ ratios for both $\operatorname{lncRNA}$ and mRNA, and accordingly comparable quality of the array data, across the board (Supplementary Fig. 1).

Scatter plots provided a profile of HUVEC IncRNAs (Fig. 1A) and mRNAs (Fig. 1B) that were upregulated, downregulated or unaffected by exposure to a high vs. normal glucose environment. Overall, the average fold changes of lncRNAs and mRNAs differentially expressed under the two study conditions were similar (Fig. 1C and 1D). Subsequent volcano plot filtering uncovered 100 significantly upregulated and 186 significantly downregulated lncRNAs in HUVECs cultured in high glucose media relative to expression in those maintained in media with normal glucose levels (Fig. $1 \mathrm{E} ; P<0.05$ ). LncRNAs that demonstrated the greatest differences in expression ranged from $171 \mathrm{bp}$ to $62.7 \mathrm{~kb}$ and are detailed in Table 1. Specifically, ENST00000421257 (RNA length: 825 bp, chromosome 20) was the most upregulated lncRNA and ENST00000559989 (RNA length: $7790 \mathrm{bp}$, chromosome 15) the most downregulated in HUVECs subjected to high vs. normal glucose culture conditions. Elevated glucose-associated changes were also noted amongst 299 mRNAs with 133 upregulated and 166 downregulated (Fig. 1F; $P<0.05$ ). qPCR conducted on 5 select IncRNAs confirmed the trend observed with the lncRNA Array analysis (Table 2). 
Supplementary Fig. 2 shows the dendrograms generated for hierarchical analysis of clustered IncRNAs and mRNAs that were differentially expressed in HUVECs cultured in media with high vs. normal glucose. Although glucose-sensitive lncRNAs were found in every human chromosome, most were located on chromosomes 1, 11 and 17 (Fig. 2A). Further probing revealed that while these differentially expressed lncRNAs are expressed along the entire length of the chromosomes (Fig. 2B), there is a notable abundance in lincRNAs (Fig. 2C).

We conducted additional profiling and found that 28 lincRNAs that were differentially expressed in response to elevated glucose are in close proximity $(<300 \mathrm{~kb})$ to 26 similarly regulated mRNAs. The fold change calculated for each lincRNA and its associated mRNA is summarized in Fig. 3A. Of note, 15 of the 28 lincRNAs demonstrated a direct correlation in fold change with its associated mRNA, whereas the remaining 13 displayed an inverse correlation.

Pathway analysis with the current KEGG database yielded several pertinent findings (Fig. 3B). In brief, lncRNAs upregulated in response to elevated glucose are involved in axon guidance while those downregulated have known associations with vascular smooth muscle cell contraction, dopaminergic synaptic signaling, ubiquitin mediated proteolysis, adrenergic signaling in cardiomyocytes, insulin signaling, and apoptosis. Table 3 lists the biological pathways that have been linked with the mRNAs found to be differentially expressed in HUVECs cultured in media with elevated vs. normal glucose.

GO analysis grouped the differentially expressed mRNAs under the following three categories: Biological Processes, Cellular Component, and Molecular Function (Table 3). GO terms most appreciably associated with differentially enriched mRNAs were cellular and 
metabolic processes (Biological Processes); cell part, cell, intracellular part; and intracellular (Cellular Component); and binding and protein binding (Molecular Function). 


\section{DISCUSSION}

This is the first report providing a complete global profile of glucose-regulated human endothelial cell-expressed lncRNAs and mRNAs. Specifically, our transcriptome survey revealed that the expression of 286 IncRNAs and 299 mRNAs were significantly different in HUVECs exposed to a high vs. normal glucose environment for 24 hours. Amongst the differentially expressed mRNAs, 26 were associated with 28 differentially expressed lincRNAs. The most upregulated mRNAs were commonly associated with axon guidance signaling pathways while those most downregulated were associated with vascular smooth muscle cell contraction, dopaminergic signaling, ubiquitin-mediated proteolysis, and adrenergic signaling networks.

Contrary to the tenet that protein-coding genes form the sole basis of gene regulation, growing evidence positions mutated lncRNAs and dysregulated lncRNA function within the scaffold of a wide range of diseases (Wahlestedt 2013; Wapinski and Chang 2011). Previous reports contend that the expression of some lncRNAs, arbitrarily defined as non-coding RNAs greater than 200 nucleotides in length (Ma et al. 2013), fluctuates with transitions from a euglycemic to hyperglycemic environment (Fadista et al. 2014; Moran et al. 2012; Yan et al. 2015). Others have shown that insulin and insulin-like growth factors transiently downregulated nuclear transcripts of the lncRNA colorectal neoplasia differentially expressed (CRNDE) gene in human colorectal and MCF-7 breast cancer cell lines (Ellis et al. 2014). Notwithstanding the morphological and functional differences between human and rodent islets and the weak conservation of human IncRNAs in rodent and in vitro models, it is worth noting that the compromised pancreatic islet structure and function observed in murine gestational diabetes were attributed to 
dysregulated lncRNA H19 (Ding et al. 2012); 303 lncRNAs were aberrantly expressed in the retinas of mice with early diabetic retinopathy (Yan et al. 2014); and the evolutionary well conserved lncRNA metastasis-associated lung adenocarcinoma transcript 1 (MALAT1) was appreciably upregulated in RF/6A choroidal endothelial cells previously exposed to high glucose (Yan et al. 2014). The results described herein therefore extend these earlier findings that support a lncRNA-diabetes relationship and also provide a potential molecular account for why endothelial dysfunction often presents in the metabolic milieu of diabetes (Booth et al. 2006).

Sequence analysis indicated that lincRNAs had a dominant presence amongst the differentially expressed IncRNAs. Although intergenic regions are robustly enriched in disease-associated polymorphisms ( $\mathrm{Li}$ et al. 2014), whether any, some or all of this subgroup of lincRNAs have functional relevance under altered glucose conditions and diabetes remains to be confirmed. Interestingly, expression associations were only detected for 28 of the 170 differentially expressed lincRNAs and with 26 differentially expressed mRNAs. This is not a surprising finding given that our knowledge of the biological significance, mechanism(s) of action, and target(s) of lncRNAs have not kept pace with our identification of novel lncRNAs. That said, our observations that the expression of 13 of the aforementioned lincRNAs directly correlated with that of their differentially expressed "partner" mRNA while an inverse correlation was noted for the remaining 15 lincRNAs and their associated protein-coding transcript is consistent with the understanding that lncRNAs both positively and negatively regulate gene expression (Wahlestedt 2013). 
Similar to Yan et al. who worked with RF/6A choroidal endothelial cells (Yan et al. 2014), Puthanveetil et al. reported significantly higher MALAT1 transcripts in HUVECs incubated with $25 \mathrm{mmol} / \mathrm{L}$ D-glucose vs. $25 \mathrm{mmol} / \mathrm{L} \mathrm{L}$-glucose (osmotic control) and 5 mmol/L glucose (normal glucose) (Puthanveetil et al. 2015). However, while MALAT1 levels increased with time in the former study, the rise was transient in the latter peaking at the 12-hour incubation mark. Whether the contrasting outcomes were due to the different cell types studied or the different concentration of glucose (50 vs. $25 \mathrm{mmol} / \mathrm{L}$ ) applied remains to be determined. Unlike these investigators, we did not detect a difference in MALAT1 expression between our normal and high glucose-treated HUVECs. Our experimental set up was the same as that of Puthanveetil's group but as we only harvested samples at the 24-hour point, we are unable to speak to the potential temporal changes in MALAT1 levels that may have also occurred in our HUVEC samples. Regardless, the collective evidence would appear to suggest that high glucose-regulated MALAT1 expression is time-dependent.

KEGG analysis revealed significant downregulation of coding transcripts that are involved in the ubiquitin-proteasomal system (UPS), a tightly controlled machinery involved in intracellular protein degradation and turnover (Jung et al. 2009). These findings are in line with an earlier report demonstrating predominant downregulation of genes of the UPS in pancreatic islets from individuals with type 2 diabetes vs. without diabetes (Bugliani et al. 2013). That deubiquitinating enzyme ubiquitin specific protease 7 is critically involved in stabilizing insulin receptor substrate (IRS)-1 and IRS-2, key mediators of insulin/insulin-like growth factor signaling, (Yoshihara et al. 2012) lends further credence to the concept of a modulatory relationship existing between the UPS and 
proteins involved with glycemia control. Indeed, pathway analysis also detected decreased expression of the calcium channel voltage-dependent, L type $\alpha 1 \mathrm{C}$ subunit $(C A C N A 1 C)$ which in mice is critical for insulin secretion (Barg et al. 2002). In human islets and $\beta$-cells, however, CACNA1C mRNA molecules were present at much lower levels than CACNA1D transcripts although there was a trend toward lower CACNA1C expression in the islets from patients with type 2 diabetes vs. without the disease (Reinbothe et al. 2013). Accordingly, whether or not $C A C N A 1 C$ plays a role in influencing insulin secretion or the insulin network in humans remains unknown.

CACNA1C, however, also encodes voltage-dependent L-type $\mathrm{Ca}^{2+}$ channels (CaV1.2) which serve as the primary $\mathrm{Ca}^{2+}$ entry pathway in vascular smooth muscle cells (Bannister et al. 2013). It may therefore regulate vasomotor tone independent of its potential metabolic effects.

The present transcriptome analysis found that several genes involved in vascular smooth muscle contraction AGEs can act via a variety of routes to propagate diabetesassociated vascular complications. On the one hand, they may amass in the extracellular space and within the vessel wall to induce oxidative stress and ramp up the atherosclerosis process (Basta et al. 2004). On the other, they may promote endothelial (progrenitor) cell apoptosis (Lan et al. 2015; Shen et al. 2010). Consistent with this, we found that the apoptosis inhibitors BIRC7 and BIRC8, were both downregulated in HUVECs that had been subjected to a high glucose environment. Taken together, these data suggest that lncRNAs may exert vascular protection by downregulating anti-apoptotic genes that participate in AGE-dependent cell demise. 
The nervous and circulatory systems are closely intertwined both anatomically and functionally, and it has been proposed that axon and capillary sprouting may be more alike than dissimilar (Klagsbrun and Eichmann 2005). Indeed, several proteins that are known to mediate axon guidance have since been determined to also be intimately involved with angiogenic processes (Klagsbrun and Eichmann 2005). Our bioinformatic analysis indicate an upregulation of genes involved with axon guidance. Given the potential premise of axons and capillaries sharing the same branching prompts, the enhanced levels of axon guidancerelated genes in HUVECs exposed to a high glucose milieu may account for the pathogenic angiogenesis typically observed in proliferative diabetic retinopathy.

Although our study is limited by the relatively low number of replicates $(n=3)$ and the reliance on a single endothelial cell type (HUVECs), we provide the first transcriptome analysis identifying endothelial lncRNAs that are differentially expressed following high glucose exposure. Inasmuch as clinical practice guidelines are unified in emphasizing the importance of early and aggressive glucose-lowering strategies to slow the development and progression of vascular complications (American Diabetes Association 2015; Canadian Diabetes Association Clinical Practice Guidelines Expert Committee 2013), these data may provide critical insights into putative biomarkers and therapeutic targets that could pave the way for novel strategies to complement the currently available vascular protective armamentarium for diabetes. 


\section{FUNDING}

This work was supported by in part by grants from the Canadian Institutes of Health Research and Heart and Stroke Foundation of Canada to S. Verma. L-E. Mantella is supported by the Canada Graduate Scholarships-Master's (CGS M) Program. S. Verma is the Canada Research Chair in Atherosclerosis at the University of Toronto.

\section{DISCLOSURES}

Nothing to disclose. No relevant relationship with industry exists.

\section{CONFLICT OF INTEREST}

None declared. 


\section{References}

American Diabetes Association. 2015. Standards of medical care in diabetes--2015. Diabetes Care, 38 Suppl: S1-93.

Avogaro, A., Albiero, M., Menegazzo, L., de Kreutzenberg, S., and Fadini, G.P. 2011. Endothelial dysfunction in diabetes: the role of reparatory mechanisms. Diabetes Care, 34 Suppl 2: S285-290. doi: 10.2337/dc11-s239.

Bannister, J.P., Leo, M.D., Narayanan, D., Jangsangthong, W., Nair, A., Evanson, K.W., Pachuau, J., Gabrick, K.S., Boop, F.A., and Jaggar, J.H. 2013. The voltage-dependent L-type $\mathrm{Ca} 2+(\mathrm{CaV} 1.2)$ channel C-terminus fragment is a bi-modal vasodilator. J. Physiol. 591(Pt 12): 2987-2998. doi: 10.1113/jphysiol.2013.251926.

Barg, S., Eliasson, L., Renstrom, E., and Rorsman, P. 2002. A subset of 50 secretory granules in close contact with L-type Ca2+ channels accounts for first-phase insulin secretion in mouse beta-cells. Diabetes, 51 Suppl 1: S74-82.

Basta, G., Schmidt, A.M., and De Caterina, R. 2004. Advanced glycation end products and vascular inflammation: implications for accelerated atherosclerosis in diabetes. Cardiovasc. Res. 63(4): 582-592. doi: 10.1016/j.cardiores.2004.05.001.

Booth, G.L., Kapral, M.K., Fung, K., and Tu, J.V. 2006. Relation between age and cardiovascular disease in men and women with diabetes compared with non-diabetic people: a population-based retrospective cohort study. Lancet, 368(9529): 29-36. doi: 10.1016/S0140-6736(06)68967-8.

Bugliani, M., Liechti, R., Cheon, H., Suleiman, M., Marselli, L., Kirkpatrick, C., Filipponi, F., Boggi, U., Xenarios, I., Syed, F., Ladriere, L., Wollheim, C., Lee, M.S., and Marchetti, P. 2013. Microarray analysis of isolated human islet transcriptome in type 2 diabetes and the role of 
the ubiquitin-proteasome system in pancreatic beta cell dysfunction. Mol. Cell Endocrinol. 367(1-2): 1-10. doi: 10.1016/j.mce.2012.12.001.

Canadian Diabetes Association Clinical Practice Guidelines Expert Committee. 2013. Canadian Diabetes Association 2013 clinical practice guidelines for the prevention and management of diabetes in Canada. Can. J. Diabetes. 37 Suppl 1: S1-212.

Chen, H.H., Almontashiri, N.A., Antoine, D., and Stewart, A.F. 2014. Functional genomics of the 9p21.3 locus for atherosclerosis: clarity or confusion? Curr. Cardiol. Rep. 16(7): 502. doi: $10.1007 / s 11886-014-0502-7$.

Chew, G.L., Pauli, A., Rinn, J.L., Regev, A., Schier, A.F., and Valen, E. 2013. Ribosome profiling reveals resemblance between long non-coding RNAs and 5' leaders of coding RNAs. Development, 140(13): 2828-2834. doi: 10.1242/dev.098343.

Cooper, M.E., and El-Osta, A. 2010. Epigenetics: mechanisms and implications for diabetic complications. Circ. Res. 107(12): 1403-1413. doi: 10.1161/CIRCRESAHA.110.223552.

Ding, G.L., Wang, F.F., Shu, J., Tian, S., Jiang, Y., Zhang, D., Wang, N., Luo, Q., Zhang, Y., Jin, F., Leung, P.C., Sheng, J.Z., and Huang, H.F. 2012. Transgenerational glucose intolerance with Igf2/H19 epigenetic alterations in mouse islet induced by intrauterine hyperglycemia. Diabetes, 61(5): 1133-1142. doi: 10.2337/db11-1314.

Ellis, B.C., Graham, L.D., and Molloy, P.L. 2014. CRNDE, a long non-coding RNA responsive to insulin/IGF signaling, regulates genes involved in central metabolism. Biochim. Biophys. Acta. 1843(2): 372-386. doi: 10.1016/j.bbamcr.2013.10.016.

Fadista, J., Vikman, P., Laakso, E.O., Mollet, I.G., Esguerra, J.L., Taneera, J., Storm, P., Osmark, P., Ladenvall, C., Prasad, R.B., Hansson, K.B., Finotello, F., Uvebrant, K., Ofori, J.K., Di Camillo, B., Krus, U., Cilio, C.M., Hansson, O., Eliasson, L., Rosengren, A.H., Renstrom, E., Wollheim, 
C.B., and Groop, L. 2014. Global genomic and transcriptomic analysis of human pancreatic islets reveals novel genes influencing glucose metabolism. Proc. Natl. Acad. Sci. U S A. 111(38): 13924-13929. doi: 10.1073/pnas.1402665111.

Fatica, A., and Bozzoni, I. 2014. Long non-coding RNAs: new players in cell differentiation and development. Nat. Rev. Genet. 15(1): 7-21. doi: 10.1038/nrg3606.

Giacco, F., and Brownlee, M. 2010. Oxidative stress and diabetic complications. Circ. Res. 107(9): 1058-1070. doi: 10.1161/CIRCRESAHA.110.223545.

Hangauer, M.J., Vaughn, I.W., and McManus, M.T. 2013. Pervasive transcription of the human genome produces thousands of previously unidentified long intergenic noncoding RNAs. PLoS Genet. 9(6): e1003569. doi: 10.1371/journal.pgen.1003569.

Hayward, R.A., Reaven, P.D., Wiitala, W.L., Bahn, G.D., Reda, D.J., Ge, L., McCarren, M., Duckworth, W.C., Emanuele, N.V., and Investigators, V. 2015. Follow-up of glycemic control and cardiovascular outcomes in type 2 diabetes. N. Engl. J. Med. 372(23): 2197-2206. doi: 10.1056/NEJMoa1414266.

Holman, R.R., Paul, S.K., Bethel, M.A., Matthews, D.R., and Neil, H.A. 2008. 10-year follow-up of intensive glucose control in type 2 diabetes. N. Engl. J. Med. 359(15): 1577-1589. doi: 10.1056/NEJMoa0806470.

Jung, T., Catalgol, B., and Grune, T. 2009. The proteasomal system. Mol. Aspects Med. 30(4): 191-296. doi: 10.1016/j.mam.2009.04.001.

Klagsbrun, M., and Eichmann, A. 2005. A role for axon guidance receptors and ligands in blood vessel development and tumor angiogenesis. Cytokine Growth Factor Rev. 16(4-5): 535-548. doi: 10.1016/j.cytogfr.2005.05.002. 
Lan, K.C., Chiu, C.Y., Kao, C.W., Huang, K.H., Wang, C.C., Huang, K.T., Tsai, K.S., Sheu, M.L., and Liu, S.H. 2015. Advanced glycation end-products induce apoptosis in pancreatic islet endothelial cells via NF-kappaB-activated cyclooxygenase-2/prostaglandin E2 upregulation. PLoS One. 10(4): e0124418. doi: 10.1371/journal.pone.0124418.

Li, X., Wu, Z., Fu, X., and Han, W. 2014. IncRNAs: insights into their function and mechanics in underlying disorders. Mutat. Res. Rev. Mutat. Res. 762: 1-21. doi: 10.1016/j.mrrev.2014.04.002.

Ma, L., Bajic, V.B., and Zhang, Z. 2013. On the classification of long non-coding RNAs. RNA Biol. 10(6): 925-933. doi: 10.4161/rna.24604.

Moran, I., Akerman, I., van de Bunt, M., Xie, R., Benazra, M., Nammo, T., Arnes, L., Nakic, N., Garcia-Hurtado, J., Rodriguez-Segui, S., Pasquali, L., Sauty-Colace, C., Beucher, A., Scharfmann, R., van Arensbergen, J., Johnson, P.R., Berry, A., Lee, C., Harkins, T., Gmyr, V., Pattou, F., Kerr-Conte, J., Piemonti, L., Berney, T., Hanley, N., Gloyn, A.L., Sussel, L., Langman, L., Brayman, K.L., Sander, M., McCarthy, M.I., Ravassard, P., and Ferrer, J. 2012. Human beta cell transcriptome analysis uncovers lncRNAs that are tissue-specific, dynamically regulated, and abnormally expressed in type 2 diabetes. Cell Metab. 16(4): 435-448. doi: 10.1016/j.cmet.2012.08.010.

Puthanveetil, P., Chen, S., Feng, B., Gautam, A., and Chakrabarti, S. 2015. Long non-coding RNA MALAT1 regulates hyperglycaemia induced inflammatory process in the endothelial cells. J. Cell Mol. Med. 19(6): 1418-1425. doi: 10.1111/jcmm.12576.

Reinbothe, T.M., Alkayyali, S., Ahlqvist, E., Tuomi, T., Isomaa, B., Lyssenko, V., and Renstrom, E. 2013. The human L-type calcium channel Cav1.3 regulates insulin release and 
polymorphisms in CACNA1D associate with type 2 diabetes. Diabetologia, 56(2): 340-349. doi: $10.1007 / s 00125-012-2758-z$.

Rinn, J.L., Kertesz, M., Wang, J.K., Squazzo, S.L., Xu, X., Brugmann, S.A., Goodnough, L.H., Helms, J.A., Farnham, P.J., Segal, E., and Chang, H.Y. 2007. Functional demarcation of active and silent chromatin domains in human HOX loci by noncoding RNAs. Cell. 129(7): 13111323. doi: 10.1016/j.cell.2007.05.022.

Shaw, A., Doherty, M.K., Mutch, N.J., MacRury, S.M., and Megson, I.L. 2014. Endothelial cell oxidative stress in diabetes: a key driver of cardiovascular complications? Biochem. Soc. Trans. 42(4): 928-933. doi: 10.1042/BST20140113.

Shen, C., Li, Q., Zhang, Y.C., Ma, G., Feng, Y., Zhu, Q., Dai, Q., Chen, Z., Yao, Y., Chen, L., Jiang, Y., and Liu, N. 2010. Advanced glycation endproducts increase EPC apoptosis and decrease nitric oxide release via MAPK pathways. Biomed. Pharmacother. 64(1): 35-43. doi: 10.1016/j.biopha.2009.03.002.

Wahlestedt, C. 2013. Targeting long non-coding RNA to therapeutically upregulate gene expression. Nat. Rev. Drug Discov. 12(6): 433-446. doi: 10.1038/nrd4018.

Wapinski, O., and Chang, H.Y. 2011. Long noncoding RNAs and human disease. Trends Cell Biol. 21(6): 354-361. doi: 10.1016/j.tcb.2011.04.001.

Yan, B., Tao, Z.F., Li, X.M., Zhang, H., Yao, J., and Jiang, Q. 2014. Aberrant expression of long noncoding RNAs in early diabetic retinopathy. Invest Ophthalmol. Vis. Sci. 55(2): 941-951. doi: 10.1167/iovs.13-13221.

Yan, B., Yao, J., Liu, J.Y., Li, X.M., Wang, X.Q., Li, Y.J., Tao, Z.F., Song, Y.C., Chen, Q., and Jiang, Q. 2015. IncRNA-MIAT regulates microvascular dysfunction by functioning as a competing endogenous RNA. Circ. Res. 116(7): 1143-1156. doi: 10.1161/CIRCRESAHA.116.305510. 
Yoshihara, H., Fukushima, T., Hakuno, F., Saeki, Y., Tanaka, K., Ito, A., Yoshida, M., Iemura, S., Natsume, T., Asano, T., Chida, K., Girnita, L., and Takahashi, S. 2012. Insulin/insulin-like growth factor (IGF) stimulation abrogates an association between a deubiquitinating enzyme USP7 and insulin receptor substrates (IRSs) followed by proteasomal degradation of IRSs. Biochem. Biophys. Res. Commun. 423(1): 122-127. doi: 10.1016/j.bbrc.2012.05.093. 


\section{Table 1}

Most differentially expressed lncRNAs in HUVECs cultured in elevated vs. normal glucose conditions

\begin{tabular}{|c|c|c|c|c|c|}
\hline & Sequence Name & $\begin{array}{c}\text { RNA } \\
\text { Length }\end{array}$ & Chromosome & Fold & $\boldsymbol{P}$ \\
\hline \multirow{10}{*}{ 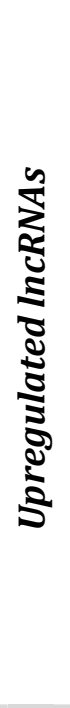 } & ENST00000421257 & 825 & 20 & 3.8073319 & 0.0238969 \\
\hline & TCONS_00007280 & 777 & 3 & 3.4405895 & 0.0373811 \\
\hline & TCONS_00016146 & 460 & 9 & 3.4268607 & 0.0383556 \\
\hline & ENST00000547721 & 779 & 12 & 3.2655793 & 0.0185213 \\
\hline & NR_047512 & 2267 & 11 & 3.2218557 & 0.0419678 \\
\hline & TCONS_00008208 & 291 & 4 & 3.0359229 & 0.0168951 \\
\hline & TCONS_00019769 & 254 & 11 & 3.0282746 & 0.0050136 \\
\hline & ENST00000565041 & 545 & 16 & 2.9479401 & 0.0134413 \\
\hline & ENST00000421891 & 831 & 6 & 2.9340224 & 0.0356850 \\
\hline & NR_024091 & 3544 & 11 & 2.8919421 & 0.04260510 \\
\hline \multirow{10}{*}{ 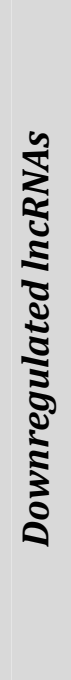 } & ENST00000559989 & 7790 & 15 & 11.1682717 & 0.0041190 \\
\hline & ENST00000458351 & 1269 & 2 & 8.0587365 & 0.0467283 \\
\hline & uc010zan.1 & 2070 & 2 & 7.2847266 & 0.0477430 \\
\hline & ENST00000465390 & 517 & 21 & 6.9831873 & 0.0492896 \\
\hline & ENST00000538550 & 553 & 11 & 6.6305 & 0.0483391 \\
\hline & TCONS_00015989 & 218 & 9 & 5.9731484 & 0.0420295 \\
\hline & TCONS_00000891 & 236 & 1 & 5.8343598 & 0.0164555 \\
\hline & uc001vvu.3 & 1388 & 14 & 5.6585137 & 0.0378370 \\
\hline & ENST00000432411 & 447 & 21 & 5.3563798 & 0.0460850 \\
\hline & uc001yvq.2 & 3358 & 15 & 5.139614 & 0.0160024 \\
\hline
\end{tabular}




\section{Table 2}

Comparison of lncRNA array and qPCR results for select lncRNAs in HUVECs cultured in elevated vs. normal glucose conditions

\begin{tabular}{|c|c|c|c|c|}
\hline Sequence Name & $\begin{array}{c}\text { Primer } \\
\text { Sequences }\left(5^{\prime}-3^{\prime}\right)\end{array}$ & $\begin{array}{c}\text { Fold } \\
\text { (lncRNA array) }\end{array}$ & $\begin{array}{c}\text { Fold } \\
\text { (validation qPCR) }\end{array}$ & p-value \\
\hline TCONS_00007280 & $\begin{array}{l}\text { F-gcagttgccatccattagc } \\
\text { R-cctccaggtggtttttaca }\end{array}$ & 3.44 & 2.83 & 0.041 \\
\hline NR_047512 & $\begin{array}{l}\text { F-cttctgctgccacatgttct } \\
\text { R-ggcctggaccagttcctc }\end{array}$ & 3.22 & 2.31 & 0.043 \\
\hline TCONS_00008208 & $\begin{array}{l}\text { F-gctgagctccttgctaatgg } \\
\text { R-ggtagcttgagtcattgtgaggt }\end{array}$ & 3.04 & 3.73 & 0.006 \\
\hline ENST00000421891 & $\begin{array}{l}\text { F-gtctcctgcgtgctcacc } \\
\text { R-cttggcttttcaggcagttac }\end{array}$ & 2.93 & 1.61 & 0.013 \\
\hline NR_024091 & $\begin{array}{l}\text { F-tgactgctcttacggaatcctat } \\
\text { R-cggtcagctcactccactg }\end{array}$ & 2.89 & 3.37 & 0.039 \\
\hline
\end{tabular}

$\mathrm{F}$ and $\mathrm{R}$ denote the forward and reverse primers, respectively. 


\section{Table 3}

Results of bioinformatic analyses on lncRNAs and mRNAs from HUVECs cultured in elevated vs. normal glucose conditions

\section{KEGG pathway analysis item}

Prion diseases

Vibrio cholerae infection

Autoimmune thyroid disease

Vascular smooth muscle contraction

Dopaminergic synapse

Ubiquitin mediated proteolysis

Apoptosis

Insulin secretion

Hematopoietic cell lineage
Count

3 C6, NCAM1, PRKACB

3 ATP6V1G2, ATP6V1H, PRKACB

3 HLA-F, IL5, TSHB

4 CACNA1C, NPPC, PLA2G1B, PRKACB

4 CACNA1C, DRD3, PPP2R5D, PRKACB

$4 \quad$ BIRC7, BIRC8, FBXO4, KLHL13

3 BIRC7, BIRC8, PRKACB

3 CACNA1C, GCG, PRKACB

3 CD33, CR1, IL5

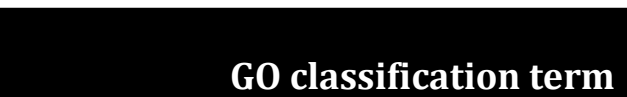

GO classification term

Cellular process

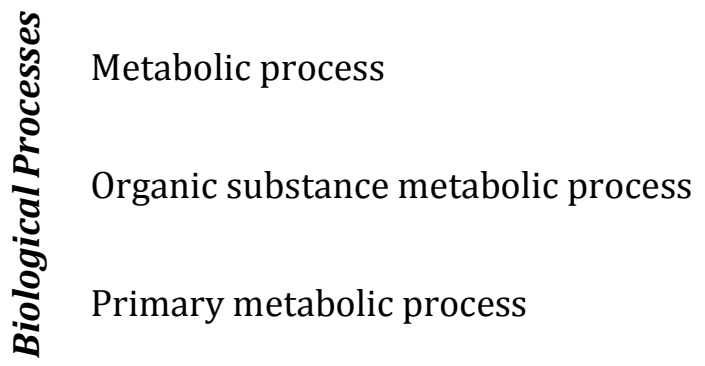

Cellular metabolic process

\section{Count}

108

107

102

99

80

\section{Proportion of differentially expressed genes}

93.10345

0.0498715

76.97842

0.032899

73.38129

0.0132884

71.22302

0.0175482

68.96552

0.0455353 
Macromolecule metabolic process

Cellular macromolecule metabolic process

Signaling

Single organism signaling

Cell communication

Cell part

Cell

Intracellular part

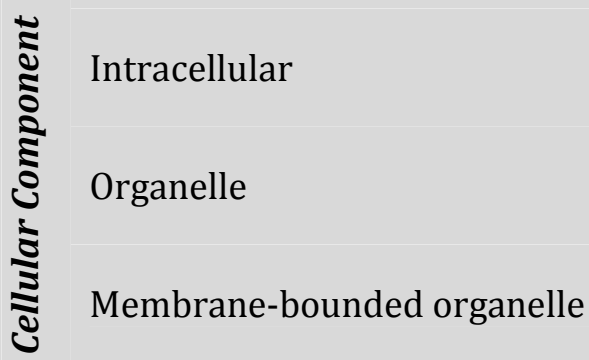

Intracellular organelle

Intracellular membrane-bounded organelle

Cytoplasm

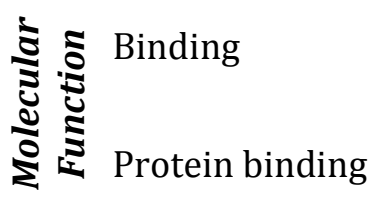

72

68

62

62

62

113

113

101

101

95

91

90

86

77

97

70
62.06897

0.0103074

58.62069

0.0027253

44.60432

0.0257421

44.60432

0.0257421

44.60432

0.0334142

94.16667

0.0090424

94.16667

0.0090848

84.16667

0.0040257

84.16667

0.0065194

79.16667

0.0166605

75.83333

0.0062574

75

0.0066086

71.66667

0.0011978

64.16667

0.0441913

88.99083

0.0096456

64.22018

0.0139664 


\section{FIGURE LEGENDS}

Fig 1. LncRNA and mRNA expression profiles in HUVECs exposed to normal (5 mmol/L) vs. high (25 mmol/L) glucose.

A, B Scatter plots comparing the variation in IncRNA and mRNA expression. The values plotted are the averaged normalized signal values (log2 scaled) for the normal glucose $(x-$ axis) and the high glucose (y-axis) groups. The green lines indicate fold change. LncRNAs and mRNAs above the top green line and below the bottom green line exhibit at least a 2.0fold difference between the two study groups. C, D Box-and-Whisker plots $\left(10^{\text {th }}, 90^{\text {th }}\right.$ percentile) showing average fold change of lncRNAs and mRNAs. Median intensity is denoted with $\mathrm{a} \rightarrow$; mean intensity with $\mathbf{a}+$. E, F Volcano plots detailing magnitude of expression difference. The vertical green lines correspond to 2.0-fold upregulation and 2.0fold downregulation of expression. The horizontal green line indicates a $P$ value of 0.05 . Red points represent lncRNAs with statistically significant differential expression (fold change $\geq$ $2.0, P \leq 0.05)$.

Fig 2. Distribution, location and classification of lncRNAs differentially expressed in HUVECs exposed to normal (5 mmol/L) vs. high (25 mmol/L) glucose.

A Chromosomal distribution. B Chromosomal location. C LncRNA classification.

Fig 3. Network co-expression and bioinformatics analyses of samples from HUVECs exposed to normal (5 mmol/L) vs. high (25 mmol/L) glucose. 
A LincRNAs and associated protein-coding transcripts. B Pathway analysis of differentially expressed protein-coding genes. Red bars denote those associated with downregulated mRNAs; green bars denote those associated with upregulated mRNAs. 
A

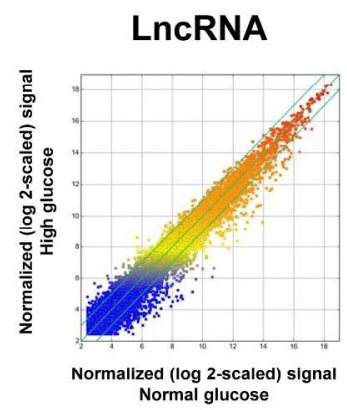

C

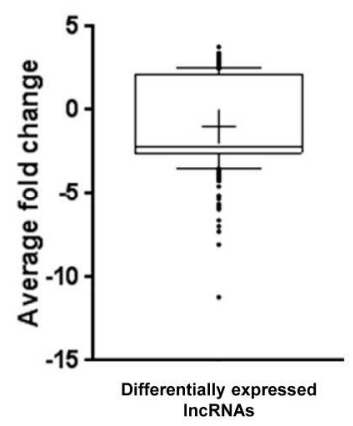

E

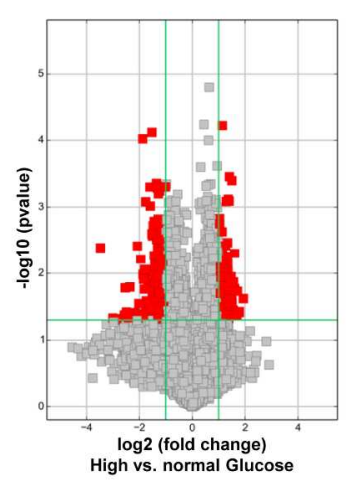

B

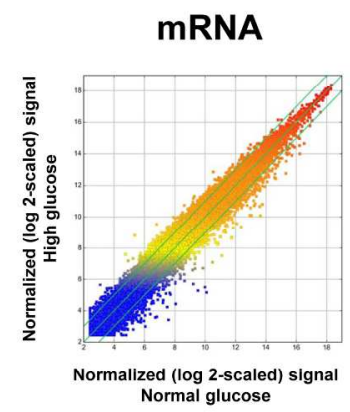

D

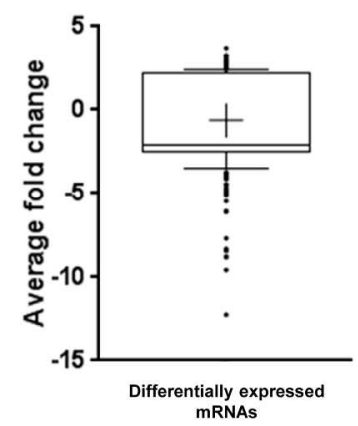

$\mathbf{F}$

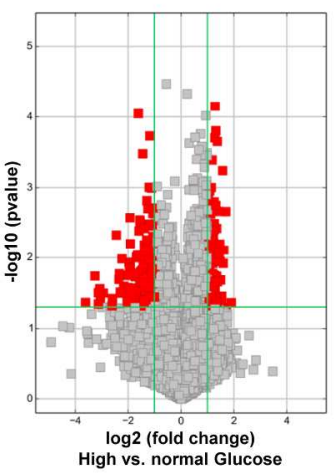

Figure 1 
Figure 2

A

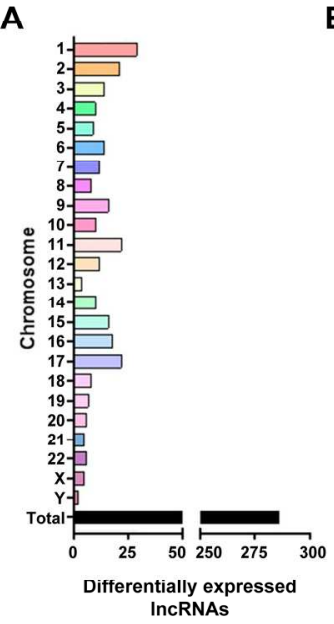

B

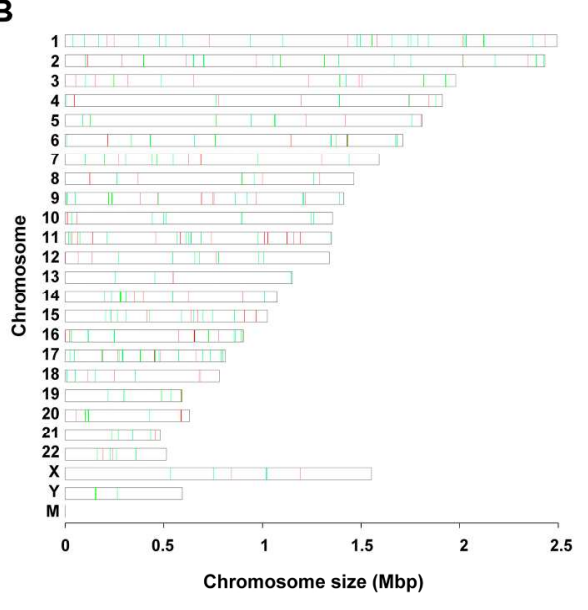

C

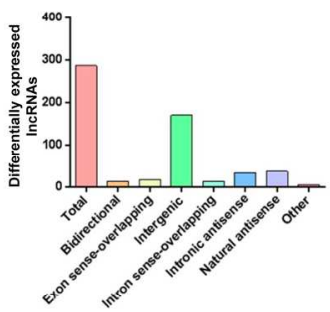

Fig 2. Distribution, location and classification of IncRNAs differentially expressed in HUVECs exposed to normal $(5 \mathrm{mmol} / \mathrm{L})$ vs. high $(25 \mathrm{mmol} / \mathrm{L})$ glucose.

A Chromosomal distribution. B Chromosomal location. C LncRNA classification.

$225 \times 168 \mathrm{~mm}(300 \times 300 \mathrm{DPI})$ 
Figure 3

A

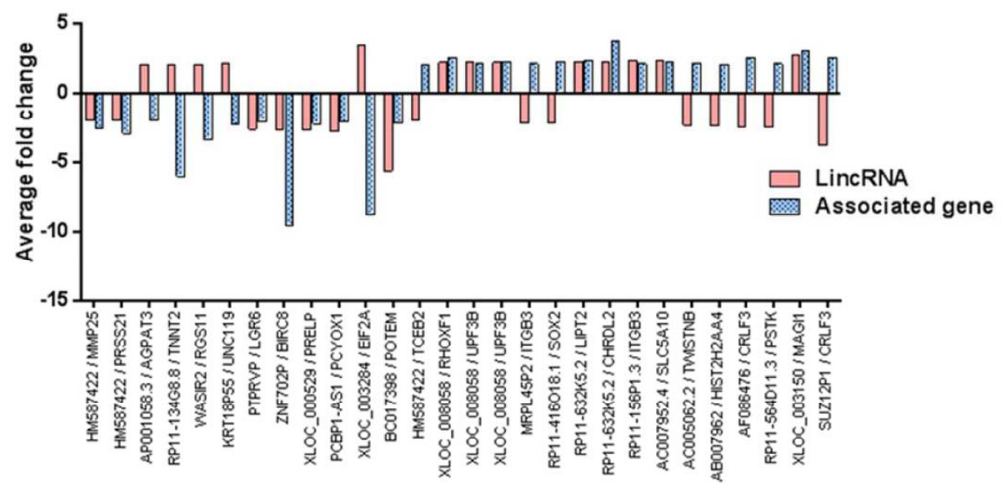

B

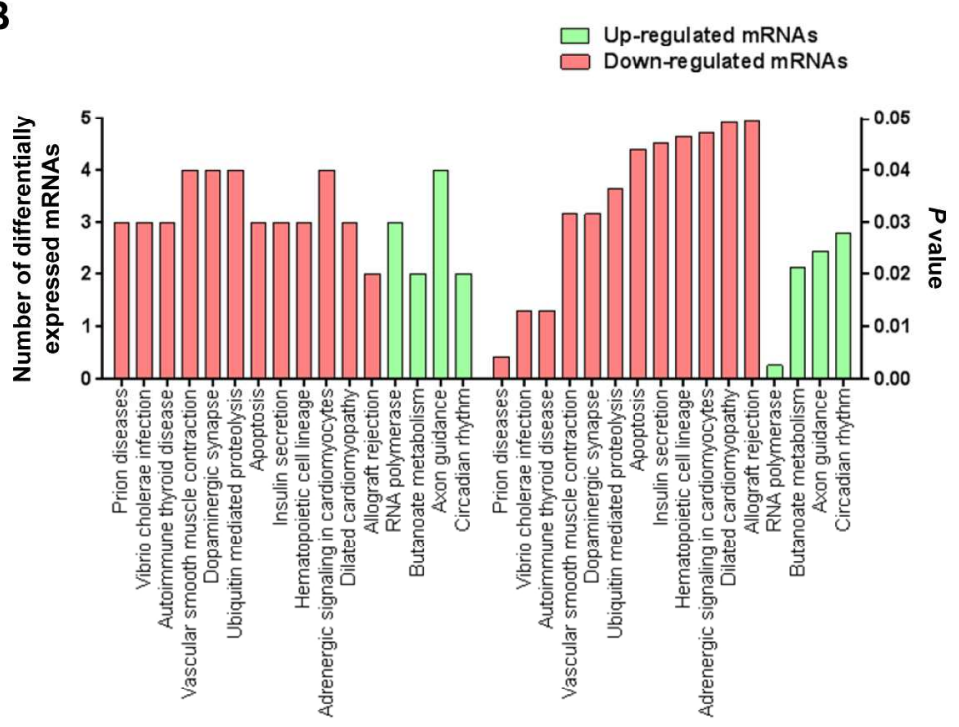

Fig 3. Network co-expression and bioinformatics analyses of samples from HUVECs exposed to normal (5 $\mathrm{mmol} / \mathrm{L})$ vs. high $(25 \mathrm{mmol} / \mathrm{L})$ glucose.

A LincRNAs and associated protein-coding transcripts. B Pathway analysis of differentially expressed proteincoding genes. Red bars denote those associated with downregulated mRNAs; green bars denote those associated with upregulated mRNAs.

$190 \times 254 \mathrm{~mm}(300 \times 300$ DPI $)$ 